\title{
The nectar report: Quantitative review of nectar sugar concentrations offered by bee visited flowers in agricultural and non-agricultural landscapes
}

\author{
Tobias Pamminger ${ }^{\text {Corresp., }}{ }^{1}$, Roland Becker ${ }^{1}$, Sophie Himmelreich ${ }^{1}$, Christof W Schneider ${ }^{1}$, Matthias Bergtold \\ ${ }^{1}$ Global Ecotoxicology, BASF SE, Limburgerhof, Germany \\ Corresponding Author: Tobias Pamminger \\ Email address: tobias.pamminger@basf.com
}

There is growing concern that some bee populations are in decline, potentially threatening pollination security in agricultural and non-agricultural landscapes. Among the numerous causes associated with this trend, nutritional stress resulting from a mismatch between bee nutritional needs and plant community provisioning has been suggested as one potential driver. To ease nutritional stress on bee populations in agricultural habitats, agrienvironmental protection schemes aim to provide alternative nutritional resources for bee populations during times of need. However, such efforts have focused mainly on quantity (providing flowering plants) and timing (during flower-scarce periods), while largely ignoring the quality of the offered flower resources. In a first step to start addressing this information gap, we have used literature data to compile a comprehensive geographically explicit dataset on nectar quality (i.e. total sugar concentration), offered to bees both within fields (crop and weed species) as well as outside fields (wild species) around the globe. Social bees are particularly sensitive to nectar sugar concentrations, which directly impact calorie influx into the colony and consequently their fitness making it an important resource quality marker. We find that the total nectar sugar concentrations in general do not differ between the three plant communities studied. In contrast we find increased variability in nectar quality in the wild plant community compared to crop and weed community, which is likely explained by the increased phylogenetic diversity in this category of plants. In a second step we explore the influence of local habitat on nectar quality and its variability utilizing a detailed sunflower (Helianthus annuus L.) data set and find that geography has a small, but significant influence on these parameters. In a third step we identify crop groups (genera), which provide sub-optimal nectar resources for bees and suggest high quality alternatives as potential nectar supplements. In the long term this data set could serve as a starting point to systematically collect more quality characteristics of plant provided resources to bees, which ultimately can be utilized by 
scientist, regulators, NGOs and farmers to improve the flower resources offered to bees. We hope that ultimately this data will help to ease nutritional stress for bee populations and foster a data informed discussion about pollinator conservation in modern agricultural landscapes. 
1 The nectar report: Quantitative review of nectar sugar concentrations offered by bee

2 visited flowers in agricultural and non-agricultural landscapes

3

4 Pamminger $\mathrm{T}^{\star 1}$, Becker $\mathrm{R}^{1}$, Himmelreich $\mathrm{S}^{1}$, Schneider $\mathrm{CW}^{1}$ \& Bergtold $\mathrm{M}^{1}$

51 Global Ecotoxicology, BASF SE, Limburgerhof, Germany

$6 \quad{ }^{*}$ Corresponding author

7 E-mail: tobias.pamminger@basf.com

8

9

\section{Abstract}

There is growing concern that some bee populations are in decline, potentially threatening pollination security in agricultural and non-agricultural landscapes. Among the numerous causes associated with this trend, nutritional stress resulting from a mismatch between bee nutritional needs and plant community provisioning has been suggested as one potential driver. To ease nutritional stress on bee populations in agricultural habitats, agrienvironmental protection schemes aim to provide alternative nutritional resources for bee populations during times of need. However, such efforts have focused mainly on quantity (providing flowering plants) and timing (during flower-scarce periods), while largely ignoring the quality of the offered flower resources. In a first step to start addressing this information gap, we have used literature data to compile a comprehensive geographically explicit dataset on nectar quality (i.e. total sugar concentration), offered to bees both within fields (crop and weed species) as well as outside fields (wild species) around the globe. Social bees are particularly sensitive to nectar sugar concentrations, which directly impact calorie influx into the colony and consequently their fitness making it an important resource quality marker. We find that the total nectar sugar concentrations in general do not differ between the three plant communities studied. In contrast we find increased variability in nectar quality in the wild plant community compared to crop and weed community, which is likely explained by the increased phylogenetic diversity in this category of plants. In a second step we explore the influence of local habitat on nectar 
quality and its variability utilizing a detailed sunflower (Helianthus annuus L.) data set and find that geography has a small, but significant influence on these parameters. In a third step we identify crop groups (genera), which provide sub-optimal nectar resources for bees and suggest high quality alternatives as potential nectar supplements. In the long term this data set could serve as a starting point to systematically collect more quality characteristics of plant provided resources to bees, which ultimately can be utilized by scientist, regulators, NGOs and farmers to improve the flower resources offered to bees. We hope that ultimately this data will help to ease nutritional stress for bee populations and foster a data informed discussion about pollinator conservation in modern agricultural landscapes.

\section{Introduction}

Pollinators are an integral part of natural as well as agricultural ecosystems, with the majority of flowering plants relying on their ecosystem services (Ollerton, Winfree et al. 2011). Over the past decades bee pollinators have received particular attention, following the realization that some populations seem to be declining (Biesmeijer, Roberts et al. 2006, Potts, Biesmeijer et al. 2010, Ollerton, Erenler et al. 2014). While managed honey bee populations are declining in only a few geographic regions and over certain time periods (Moritz and Erler 2016), the focus of concern has recently extended to wild bees (Roulston and Goodell 2011, Goulson, Nicholls et al. 2015, Vaudo, Tooker et al. 2015). Numerous potential drivers for this proposed dynamic have been put forward including changes in land use, agricultural intensification, habitat loss- or fragmentation and emerging pathogens (Brown and Paxton 2009, Winfree, Aguilar et al. 2009, Goulson, Nicholls et al. 2015). While all these factors likely contribute to some degree, changes in flower provided food resource for bees has emerged as prime candidate directly regulating bee populations (Roulston and Goodell 2011). Bees and their larvae almost exclusively rely on flower derived nutrients, namely nectar as their primary source of carbohydrates and pollen for protein, lipids, and other micronutrients essential for development, health and survival (Michener 2000, Brodschneider and Crailsheim 2010, Roulston and Goodell 2011). Large scale changes in land-use can alter the quality, 
abundance and availability of relevant flower derived resources, which in turn can result in nutritional mismatch leading to nutritional stress for bee populations with potential adverse effects (Potts, Biesmeijer et al. 2010, Roulston and Goodell 2011, Goulson, Nicholls et al. 2015). For example, while bee pollinated crops might provide a plethora of flower derived resources during their flowering period, the lack of alternative food sources in monocultural dominated agricultural settings, might put a strain on bee species foraging outside the flowering period.

In order to ease nutritional stress on manages as well as wild bee populations in agricultural settings the establishment of complementary foraging habitats has been incentivized via agro-environmental management schemes in the EU and elsewhere (Phillips and Lowe 2005, Vaughan and Skinner 2008, Lye, Park et al. 2009, Goulson, Nicholls et al. 2015, Potts, Biesmeijer et al. 2015). Such schemes were originally intended to provide bees with complementary flower resources outside the mass flowering periods of commercial crops, but have traditionally been intended to supporting social bees i e. Bombus sp. (Vaudo, Tooker et al. 2015). Only recently the effects of nutritional enhancement on wild bees which often have different habitats and nutritional requirements have come more into focus (Scheper, Bommarco et al. 2015). Besides quantity and timing the quality of floral resources, including total sugar content and sugar concentration have direct fitness consequences for social bees (Brodschneider and Crailsheim 2010, Vaudo, Tooker et al. 2015, Vaudo, Patch et al. 2016), but likely also solitary bees at least to some degree. Consequently qualitative aspects of nectar resources should be taken into consideration when developing management plans for complementary and high quality nutritional bee resources (Vaudo, Tooker et al. 2015).

As a first step to facilitate the integration of flower resource quality in pollinator management we have used literature data to compile a geographically explicit data base of nectar quality (measured as total sugar concentration) provided by bee visited flowers in an agricultural and natural setting. Given that nectar is the main carbohydrate source for adults as well as developing bees, sugar concentration is directly linked to the amount of sugar bees can extract from flowers and has traditionally served as a proxy for nectar quality (Roulston and Goodell 2011, Vaudo, Tooker et al. 2015). 
89

90

91

92

93

94

95

96

97

98

99

100

101

102

103

104

105

106

107

108

109

110

111

112

113

114

115 Geographic localization

116

117

\section{Materials and Methods}

\section{Plant selection} derived classifications.

We use the complied data base to compare the quality and quality variability of nectar resource bees can encounter in agricultural landscapes in- (crop and weeds) and off-field (wild) around the globe. In a second step we utilize a unique historical data set to analyze the influence of local habitat and water stress on nectar sugar concentrations and their variability. In a last step we identify crop genera, which provide sub-optimal nectar quality and suggest plant groups which could be used to nutritionally support bee populations in agricultural landscapes during times of need.

Data collection and categorization

In late 2017 and early 2018 we searched the literature for records on nectar quality in bee pollinated flowers using ISI web of knowledge and google scholar. We used the search terms: flower AND nectar AND sugar concentration adding either pollinator or bee as additional search term. Using these results, we identified relevant publications by scanning the title and abstract. Based on this refined list we extended our search to the literature cited within the relevant publications. Following the first scan of the primary English literature online, we search for older German primary as well as secondary literature (Books) in our company internal library.

Plant species were categorized as bee visited if either bee pollination was directly observed or the flowers were explicitly classified as "melittophil" based on their floral characteristics by the study authors. In addition, we used the USDA pollinator manual (McGregor 1976) and the expertise of BASF plant experts for cross validation of the

We chose to map the plant distribution on a continental scale because this information was available for the majority of plant species included in the data set. We decided to 
118 choose the Panama Canal as separation line between North and South America the Ural 119 and the black sea to separate Europe from Asia and the Suez Canal to separate Asia and Africa. Using the encyclopedia of life (http://eol.org/) as source for plant distribution we recorded the presence and absence of collection records of each plant species on the five continents. This very broad geographical classification is intended as a first attempt to make this information geographically explicit and should serve as a starting point to add more detailed information on the local geographic (e g. national or region) or habitat characteristics in the future. Such information will be vital to make more precise predictions about the temporal quality dynamics in agricultural landscapes around the globe.

Categorization of crop, weed and wild plants

The selected plants were categorized as crop species if they were listed as "cultivated crops" in any of the available governmental data bases (e. g. USDA: https://plants.usda.gov and European commission plant variety catalogue: https://ec.europa.eu, (McGregor 1976)), the open primary literature or were known as such to our BASF crop experts. All remaining plants without such record were categorized as non-cultivated. In a second step these non-cultivated plants were classified either as a weed species, in case they were listed in at least one of the following agricultural or governmental weed data resource (USA Noxious weed data base https://plants.usda.gov, Australia weeds http://www.environment.gov.au or industry compendium (Bayer 1992)), or as wild plants in case they were not mentioned in one of these data bases. Once a plant species was categorized (as crop weed or wild) in one geographic region it was classified as such in all other regions where it was present.

\section{Resource quality}

144 We used sugar (total carbohydrate) concentration in nectar (\% w/w) as proxy for nectar quality. This quality characteristic was chosen because it is the most frequently reported quantitative measurement of nectar quality in the literature, and is directly related to bee fitness (Vaudo, Tooker et al. 2015). However, it is important to mention that other quality 
148 criteria (e g. sugar composition, nectar volume as well as the presence and absence of 149 non-sugar compounds) are also important markers for resource quality (Vaudo, Tooker 150 et al. 2015). In particular nectar volume is likely a secondary main driver for nectar quality, 151 combined with the sugar concentrations it determines the total caloric value per flower. 152 However, such information is scarce and was consequently not included in this project.

Nectar quality categorization

155

156

157

158

159

160

161

162

163

164

165

166

167

168

169

170

171

172

173

174

175

Nectar serves as the main carbohydrate source for bees and consequently the total caloric value as well as the rate of calorie uptake are important aspects of nectar quality for them. One of the main factors determining uptake rate is nectar viscosity, which in term is largely determined by nectar sugar concentration. Based on uptake measurements and theoretical consideration the bee optimal concentration range was determined as $35-65 \%$ (Kim, Gilet et al. 2011). While this is a theoretical optimal range and bees seem to prefer higher over lower nectar sugar concentrations (Wykes 1952, Roubik and Buchmann 1984, Cnaani, Thomson et al. 2006) they will collect nectar with sugar concentrations below that value under natural conditions (e g. (Roubik and Buchmann 1984)). However, available evidence suggests that at least social bees avoid foraging on nectar sources below $20 \%$ sugar concentration, likely because the caloric intake cannot support sustained foraging activity with potentially detrimental effects for the bee colony (Maurizio and Grafl 1980, Roubik and Buchmann 1984, Cnaani, Thomson et al. 2006). While most of these results are based on findings in social bees (honeybee and bumblebee) we can assume that most of the basic physiological limitations (energy expenditure during flight and physics of suction feeding) apply to solitary bees as well. Based on these criteria we define nectar concentrations of $65-35 \%$ as optimal $35-20 \%$ as adequate and nectar sugar concentrations below $20 \%$ as low quality.

Analysis

Nectar quality and its variability in bee visited plants 
176 In the first part of the analysis we focused on the broad picture of nectar quality and its 177 variation provided by a given plant community (crop, weeds and wild) on all relevant 178 continents around the globe. In addition, we explore the possibility of intrinsic differences 179 in nectar quality variability of the plant species belonging to the different communities 180 (crop, weed and wild) using plant species where we had multiple quality measurements $(\mathrm{N}>3)$ to calculate standard deviation (SD) as a proxy for within species variability.

The influence of local habitat on nectar quality and its variability

184

185

186

187

188

189

190

191

192

193

194

195

196

197

198

199

200

201

202

203

204

During our non-english literature screening we discovered a data set (Simidtschiev 1988), which is uniquely suited to isolate the contribution of geographic location and water availability to nectar sugar concentrations and its variation in the sunflower (Helianthus annuus L.). In order to make this data easier accessible to the scientific community, we will give a brief summary of the materials and methods used. Between 1981 and 1986 a field experiment was conducted at two field sites in Bulgaria (Toshevo in north-east and Plovdiv in central Bulgaria) separated by more than $300 \mathrm{~km}$. Over this time period 52 sunflower variants and hybrids, originating from different geographical regions around the globe (including Europe, North America, South America and Australia), were grown under standard agronomical conditions at both locations. The nectar sugar concentration for all varieties were measured each year on 25 flowers per variant/hybrid day and location (200-300 measurements per year) using a capillary based extraction method and an Abbe Refractometer. In a second experiment, the author tested the effect of irrigation (watering vs. no watering) using a subset of four varieties. Using this unique data set (Simidtschiev 1988) we explore the influence of location on nectar sugar concentration variation in the sunflower taking advantage of the paired design of the study.

Nectar quality offered by plant genera

In a last step we compared the quality of crop genera in terms of nectar quality. We used all genera, where we had measurements for more than 3 plant species belonging to this genus. We characterized the selected genera according to our pre-defined categories 
205 (see above) as optimal (35-65\%), adequate (34-20\%) and low quality (below 20\%). We 206 used this information to identify crop genera offering low quality nectar and potential 207 genera offering high nectar quality as potential replacements.

208

209 Statistics

210 Both statistical analysis and graphs generation were conducted in R v. 3.3.3. (Team 2013)

211 We used descriptive statistics, conservative non-parametric Kruskal-Wallis (KW) and the 212 Fligner-test (FT) to explore the overall differences between the three plant communities 213 both in terms of nectar sugar concentration (KW) and its variation (FT) on a global level 214 and within the geographic regions. In case the main test indicated significant differences, 215 a Bonferroni corrected pairwise test (KW or FT) was used. To explore differences in the 216 nectar quality variability on a species level between communities (crop, weed and wild) 217 we used a KW test. In order to test for the influence of the geographic location of cultivation (Simidtschiev 1988 data set) on nectar concentrations and its variation we used a paired Wilcoxon-test as well as a FT test. To investigate the last hypothesis of genus specific differences in nectar quality a KW test was used. This approach was chosen to present the overall patterns in nectar quality and its variation, which does not take into account the phylogenetic dependencies of the individual plant species. In case this data would be used to identify potential drivers for the observed variation a phylogenetically controlled approach would be more appropriate. However, the main focus of this paper is the presentation of the overall broad patterns, while an in depth analysis of the factors driving it were beyond the scope of this project. Significance level were set to $\alpha=0.05$ in all cases.

228

229

Results

230

Data summary

231

In total we collected 444 individual measurements of sugar concentration in nectar for bee pollinated flowers ranging from $6.3-85 \%$. With similar sampling sizes for plant species in crop $(N=151)$ and wild plants $(N=141)$, but fewer measurements for weeds 
$234(\mathrm{~N}=30)$. On a genus level we find that the wild community has the highest phylogenetic 235 diversity in terms of number of genera recorded $(\mathrm{N}=63)$ followed by the crop community $236(\mathrm{~N}=29)$ and lowest diversity in the weed community $(\mathrm{N}=18)$. In general, the recorded 237 data is evenly spread across the geographic regions (see Tab. 1), however only a limited 238 number of weed species could be identified in Africa $(N=13)$ and South America $(N=$ 239 18). The summary statistics including mean, median $10^{\text {th }}$ and $25^{\text {th }}$ percentile are 240 presented in Table 1.

242 Nectar quality \& variability

243 Overall nectar concentration in all regions were comparable around a median value of 244 $40 \%$ sugar concentration (see Figure 1, Tab.1) and no significant differences between 245 crop, weed or wild plant communities were found globally $\left(\mathrm{KW} \mathrm{chi}{ }^{2}=3.2, p=0.2\right)$ or within 246 the different geographic regions (all KW chi ${ }^{2}<4.48, p>0.11$; see Figure 1 and Tab. 1). 247 In contrast to the median concentrations we find that the three plant communities differed 248 in the variability of nectar quality (Global community; Fligner test $\mathrm{chi}^{2}=31.97, p<0.001$ ). 249 This effect is mainly driven by an increased variability of the wild community (see Fig.1) 250 which differs significantly from the crop community on a global level (Bonferroni corrected pairwise Fligner test crop $x$ wild $\mathrm{chi}^{2}=30.64, \mathrm{p}<0.001$ ), with a similar trend in the same direction when compared to the weed community (Bonferroni corrected pairwise Fligner test; crop $x$ weed $\left.\mathrm{chi}^{2}=5.02, p=0.08\right)$. In contrast we find that crop and weed species clearly do not differ in terms of their variability (Bonferroni corrected pairwise Fligner test 255 weed $x$ wild $\left.\mathrm{chi}^{2}=1.01, p=0.93\right)$. When comparing the variability of nectar quality on a species level we find that we had only a limited number of species with multiple nectar measurements $(N>2)$ recorded (crop $N=18$, weed $N=6$ and wild $N=18)$. Using this limited data set we find no indication of intrinsic difference in variability (measured as SD) of plant species belonging to the three different plant communities $\left(\mathrm{KW} \mathrm{chi}{ }^{2}=2.52, \mathrm{p}=\right.$ 260 $0.28)$. 
263 When reanalyzing the Simidtschiev (1988) sunflower dataset comparing nectar sugar 264 concentrations we find that geographic location has a small (Median $_{\text {Toschevo }}=30.7 \%$, 265 Median $_{\text {Plovdiv }}=36.05 \%$ see Fig.2), but significant influence on nectar concentrations 266 (paired Wilcoxon test: $\mathrm{V}=216, \mathrm{p}<0.0001$ see Fig.2) and its variation (Fligner test $\mathrm{chi}^{2}=$ $2676.12, p=0.01$ see Fig.2). When looking at the effect of non-irrigation (natural rainfall) on 268 nectar sugar concentration of the four tested sunflower varieties the original analysis of 269 Simidtschievs (1988) concludes that in three of the four varieties watering did not significantly influence nectar sugar concentration and in the fourth cultivar (hybrid 260) it only decreased it by about $3.4 \%$ (mean irrigation $=50.3$, mean natural rainfall $=53.7 \%$ ).

272

273

Nectar quality on a genus level

274 In total we recorded multiple measurements for 12 crop and 16 non-crop genera and find 275 that there is a significant differences in nectar sugar concentration between them (KW 276 $\mathrm{chi}^{2}=149.23, \mathrm{p}<0.0001$ Fig.3). When comparing the nectar quality according to our categorization (see above) our results indicate that two crop genera, namely Capsicum 278 (including paprika and chili) and Pyrus (pear), offer low quality nectar (median sugar

279

280

281

282

283

284

285

286

287

288

289

290

291 concentration $<20 \%$. (see Fig. 3 ). When looking at the genera offering high quality nectar we were able to identify 15 Genera which provide optimal nectar concentrations (35\%$65 \%$ ) for bees (see Fig.3).

\section{Discussion}

In this study we have compiled the first comprehensive data set on nectar quality provided by bee visited plants in agricultural landscapes around the globe. Our data indicates that nectar sugar concentrations in bee visited flowers is strongly conserved across all communities and geographic regions with a median value around $40 \%$ (see Fig. 1 and Tab.1). In addition, we find that wild plants exhibit stronger variation in nectar concentrations at the community level when compared to crop plants. However, this difference is not reflected on the species level. Using a comprehensive data set from the German literature (Simidtschiev 1988) on sunflower varieties we find evidence that 
292 microhabitat (e g. water availability) and geographic region might have a more limited 293 effect on nectar sugar concentration and its variation than previously thought. Using the 294 complete data set we identify two crop genera (Capsicum and Pyrus) which provide low 295 296 quality nectar to bees during their flowering period and suggest 15 possible genera which provide high quality nectar as potential supplement nectar source.

When looking at the recorded nectar sugar concentrations we find strong support for the well-established idea that flowers are under strong selection pressure to provide 299 nectar suitable for their respective pollinators (Baker 1975, Harder 1986, Perret, 300 Chautems et al. 2001). In the case of bees, the literature suggests optimal values ranging from $35-65 \%$ which is well supported by our data (Waller 1972, Harder 1986, Kim, Gilet 302 et al. 2011). It has been suggested that the pollinator preferences for different sugar 303 concentrations could be explained by different modes of nectar intake, which in case of 304 bees favours higher viscosity and consequently sugar concentrations (Kim, Gilet et al. 305 2011). Our results therefore indicate that nectar quality between different plant 306 communities in agricultural landscapes and their surroundings are 1) closely matched to pollinators needs 2 ) comparable between all regions and 3) in principal likely sufficient to 308 maintain healthy bee pollinator populations.

In contrast to the median sugar concentrations we find elevated nectar quality variability of the wild plant community compared to the crop and likely weed plants communities (see Fig. 1). However, based on the results of our limited species level data set we have no indication that this pattern is reflected on the species level where plants of the different communities exhibited comparable variability. In particular the species belonging to the weed community are interesting in this regard as they are wild species which grow under (invade) standardized agricultural conditions. A priori we could expect that the more standardized growing conditions in agricultural could reduce nectar quality variability when compared to natural habitats. As we do not find differences between these three groups on the species level our results support the view that growing conditions might have a more limited influence on the variability of nectar sugar concentration and supports a plant species specific nectar concentration of at least some species. In turn this suggest that the observed variation on the community level likely 
322 reflects the elevated phylogenetic diversity in wild plants compared to crop and weed 323 species (Meyer, DuVal et al. 2012). Indeed, when looking at the community level, we find 324 the wild community containing more than twice the number of wild genera $(N=63)$

325 compared to the crop $(\mathrm{N}=29)$, as well as the weed community $(\mathrm{N}=18)$, which seems the most likely explanation for the observed pattern.

The comprehensive study of Simidtschiev (1988) offers the unique opportunity to study the effects of geography and water availability on nectar sugar concentration. These results support our initial findings that geography, in this case only has a limited absolute effect on nectar sugar concentration and its variation (See Fig. 2). In particular the fact that plants "defended" their nectar sugar concentrations against variation in water availability suggest that at least sunflowers have plant species specific nectar sugar concentrations. In his original analysis Simidtschiev (1988) suggests that instead of changes in nectar concentration, nectar volume responds to reduced water availability, which in turn reduce the caloric value and consequently resource quality for bees. It would be interesting to analyses what parameters best explain the observed variation (including temperature, rainfall soil types ect.) because such factor undoubtedly play a role in shaping nectar concentrations to some degree (Corbet, Willmer et al. 1979). Unfortunately, these parameters were not recorded by Simidtschiev (1988) for the study duration. This highlights the importance of multiple measurements to adequately characterize resource quality for bees. In a next step it would be very important to include such measurements into the data base to provide a more detailed picture of nectar quality to serve as a robust basis to improve the resource quality offered to bee populations in the future.

In our quality analysis we have identified two genera of crop plants, which provide low quality nectar sugar concentrations. While it is well-known that pears (Pyrus) are not considered attractive to social bees due to their low nectar (Fig.3) quality (Maurizio and Grafl 1980) the even lower levels in Capsicum (e g. paprika and chilies) suggest that these crop will likely not be able to sustain managed bee populations on their own. Such information, coupled with detailed information regarding geographic abundance of these crops, could be used to identify potential targets for a resource quality intervention such 
352 as agro-environmental scheme. We suggest 15 plant genera which provide adequate 353 nectar quality for bees and could be used as feeding supplement. For example, pear 354 orchards might be a good option for such interventions as the supplemental nectar 355 resources could be located close by, which in turn could attract and retain social as well as solitary bees which use pear flowers as a source of pollen (Westrich 1989).

357

358

359

360

361

362

363

364

365

366

367

368

369

370

371

372

373

374

375

376

377

378

379 380

381

Our study is a first step towards the integration of resource quality in bee conservation practices. The obvious next step could be to include additional quality markers for nectar including nectar volume, sugar composition and non-sugar components, which clearly play an important role in determining nectar quality for bees (Vaudo, Tooker et al. 2015). In particular nectar volume and flowering season are promising next steps to get a more complete picture of nectar quality because together concentration and volume define the caloric value of individual flowers, entire plants and ultimate vegetation types. The caloric value of flowers is likely important for most bee species, however most studies directly linking nectar quality to bee fitness stem from social bees such as honey and bumblebees. Currently it is less clear how important these factors are for solitary bees' fitness considering their deviating ecology. Such information would be useful to specifically address the needs of solitary bees. A second important step would be to compile a similar data set for the second important flower resource pollen and its quality markers such as crude protein content, amino acid composition or lipid content (Roulston, Cane et al. 2000). Such information should be combined with detailed geographic and information on exact flowering periods in order to estimate the resource availability and quality during the season in a given location and plan and implement targeted interventions to support bee populations.

Such a tool could support farmers, scientists, regulators NGOs and the industry when designing optimized alternative flower resources for bees in agricultural landscapes. We hope that this data set will serve as a starting point to help facilitate a data informed discussion about pollinator conservation in agricultural landscapes between all relevant stakeholders and will ultimately help to reduce nutritional stress for bee populations in modern agricultural landscapes. 
382

383

384

385

386

387

388

389

390

391

392

393

394

395

396

397

398

399

400

401

402

403

404

405

406

407

408

409

410

411

412

413

414

415

416

417

418

419

420

421

422

423

424

Conclusion

In this study we conducted the first systematic review of nectar sugar quality bees can encounter in agricultural landscapes in- and off-field around the globe. We report that nectar sugar concentrations do not vary between regions or habitats with a median of around $40 \%$ nectar total sugar concentration. We have identified several crop genera providing nectar with sub optimal sugar concentrations for bees which could potentially benefit from alternative nectar sources during their mass flowering period. This dataset is only a first step toward integrating nectar sugar concentrations into bee management practices and we hope that this data resource will facilitate communication between all relevant stakeholders and ultimately help to reduce nutritional stress for bee populations in modern agricultural landscapes.

\section{References}

Baker, H. G. (1975). "Sugar concentrations in nectars from hummingbird flowers." Biotropica: 37-41. Bayer, A. (1992). "Important crops of the world and their weeds." Scientific and common names, synonyms, and WSSA/WSSJ approved computer codes. Business Group Crop Protection, Bayer AG, Leverkusen, Germany.

Biesmeijer, J. C., S. P. Roberts, M. Reemer, R. Ohlemüller, M. Edwards, T. Peeters, A. Schaffers, S. G. Potts, R. Kleukers and C. Thomas (2006). "Parallel declines in pollinators and insect-pollinated plants in Britain and the Netherlands." Science 313(5785): 351-354.

Brodschneider, R. and K. Crailsheim (2010). "Nutrition and health in honey bees." Apidologie 41(3): 278294.

Brown, M. J. and R. J. Paxton (2009). "The conservation of bees: a global perspective." Apidologie 40(3): 410-416.

Cnaani, J., J. D. Thomson and D. R. Papaj (2006). "Flower choice and learning in foraging bumblebees: effects of variation in nectar volume and concentration." Ethology 112(3): 278-285.

Corbet, S., P. Willmer, J. Beament, D. Unwin and O. Prŷs-Jones (1979). "Post-secretory determinants of sugar concentration in nectar." Plant, Cell \& Environment 2(4): 293-308.

Goulson, D., E. Nicholls, C. Botías and E. L. Rotheray (2015). "Bee declines driven by combined stress from parasites, pesticides, and lack of flowers." Science 347(6229): 1255957.

Harder, L. D. (1986). "Effects of nectar concentration and flower depth on flower handling efficiency of bumble bees." Oecologia 69(2): 309-315.

Kim, W., T. Gilet and J. W. Bush (2011). "Optimal concentrations in nectar feeding." Proceedings of the National Academy of Sciences 108(40): 16618-16621.

Lye, G., K. Park, J. Osborne, J. Holland and D. Goulson (2009). "Assessing the value of Rural

Stewardship schemes for providing foraging resources and nesting habitat for bumblebee queens (Hymenoptera: Apidae)." Biological Conservation 142(10): 2023-2032.

Maurizio, A. and I. Grafl (1980). Das Trachtpflanzenbuch.

McGregor, S. E. (1976). Insect pollination of cultivated crop plants, Agricultural Research Service, US Department of Agriculture Washington (DC).

Meyer, R. S., A. E. DuVal and H. R. Jensen (2012). "Patterns and processes in crop domestication: an historical review and quantitative analysis of 203 global food crops." New Phytologist 196(1): 29-48.

Michener, C. D. (2000). The bees of the world, JHU press. 
425

426

427

428

429

430

431

432

433

434

435

436

437

438

439

440

441

442

443

444

445

446

447

448

449

450

451

452

453

454

455

456

457

458

459

460

461

462

463

464

465

466

467

468

469

Moritz, R. F. and S. Erler (2016). "Lost colonies found in a data mine: global honey trade but not pests or pesticides as a major cause of regional honeybee colony declines." Agriculture, Ecosystems \&

Environment 216: 44-50.

Ollerton, J., H. Erenler, M. Edwards and R. Crockett (2014). "Extinctions of aculeate pollinators in Britain and the role of large-scale agricultural changes." Science 346(6215): 1360-1362.

Ollerton, J., R. Winfree and S. Tarrant (2011). "How many flowering plants are pollinated by animals?" Oikos 120(3): 321-326.

Perret, M., A. Chautems, R. Spichiger, M. Peixoto and V. Savolainen (2001). "Nectar sugar composition in relation to pollination syndromes in Sinningieae (Gesneriaceae)." Annals of Botany 87(2): 267-273.

Phillips, A. and K. W. Lowe (2005). "Prioritising integrated landscape change through rural land stewardship for ecosystem services." Australasian Journal of Environmental Management 12(sup1): 3946.

Potts, S., K. Biesmeijer, R. Bommarco, D. Kleijn and J. Scheper (2015). Status and trends of European pollinators. Key findings of the STEP project, Pensoft Publishers.

Potts, S. G., J. C. Biesmeijer, C. Kremen, P. Neumann, O. Schweiger and W. E. Kunin (2010). "Global pollinator declines: trends, impacts and drivers." Trends in ecology \& evolution 25(6): 345-353.

Roubik, D. W. and S. L. Buchmann (1984). "Nectar selection by Melipona and Apis mellifera

(Hymenoptera: Apidae) and the ecology of nectar intake by bee colonies in a tropical forest." Oecologia 61(1): 1-10.

Roulston, T. a. H., J. H. Cane and S. L. Buchmann (2000). "What governs protein content of pollen:

pollinator preferences, pollen-pistil interactions, or phylogeny?" Ecological monographs 70(4): 617-643.

Roulston, T. a. H. and K. Goodell (2011). "The role of resources and risks in regulating wild bee populations." Annual review of entomology 56: 293-312.

Scheper, J., R. Bommarco, A. Holzschuh, S. G. Potts, V. Riedinger, S. P. Roberts, M. Rundlöf, H. G. Smith, I. Steffan-Dewenter and J. B. Wickens (2015). "Local and landscape-level floral resources explain effects of wildflower strips on wild bees across four European countries." Journal of Applied Ecology 52(5): 1165-1175.

Simidtschiev, T. (1988). "Nektarproduktivität von Sonnenblumensorten,

Hybriden und Linien verschiedener Herkunft." Die biene(12): 637-641.

Team, R. C. (2013). "R: A language and environment for statistical computing."

Vaudo, A. D., H. M. Patch, D. A. Mortensen, J. F. Tooker and C. M. Grozinger (2016). "Macronutrient ratios in pollen shape bumble bee (Bombus impatiens) foraging strategies and floral preferences."

Proceedings of the National Academy of Sciences 113(28): E4035-E4042.

Vaudo, A. D., J. F. Tooker, C. M. Grozinger and H. M. Patch (2015). "Bee nutrition and floral resource restoration." Current Opinion in Insect Science 10: 133-141.

Vaughan, M. and M. Skinner (2008). "Using Farm Bill programs for pollinator conservation." USDANRCS National Plant Data Center, online: http://www. xerces. org/wpcontent/uploads/2008/11/using farm bill programs xerces society. pdf.

Waller, G. D. (1972). "Evaluating responses of honey bees to sugar solutions using an artificial-flower feeder." Annals of the Entomological Society of America 65(4): 857-862.

Westrich, P. (1989). "The wild bees of Baden-Württemberg." The wild bees of Baden-Württemberg. Winfree, R., R. Aguilar, D. P. Vázquez, G. LeBuhn and M. A. Aizen (2009). "A meta-analysis of bees' responses to anthropogenic disturbance." Ecology 90(8): 2068-2076.

Wykes, G. (1952). "The preferences of honeybees for solutions of various sugars which occur in nectar." Journal of Experimental Biology 29(4): 511-519. 


\section{Figure 1 (on next page)}

\section{Summary of the nectar sugar concentration of bee visted flowers around the globe}

Summarizes the total nectar sugar concentration [\%] in agricultural landscapes on a global as a continental level. We present data for Europe, North America, South America, Africa, Australia and overall (Global) for crop (A-G), weed and wild plant communities. Results of the statistical analysis (Kruskal Wallis (KW) $\mathrm{Chi}^{2}$, and $p$ values) are presented in the upper left corner of the individual Panels. 


\section{Figure 2 (on next page)}

Nectar sugar concentrations of 52 sunflower varieties grown at two locationnin Bulgaria

The graph depicts the nectar sugar concentration of 52 sunflower varieties (Helianthus annuus) grown at two geographic locations in Bulgaria (Toschevow and Plovdiv area) between 1981 and 1986. Data taken and re analyzed from Simidtschiev (1988). Results of the statistical analysis (paired Wilcoxon test, $V$ and $p$ values) are presented in the lower left corner. 


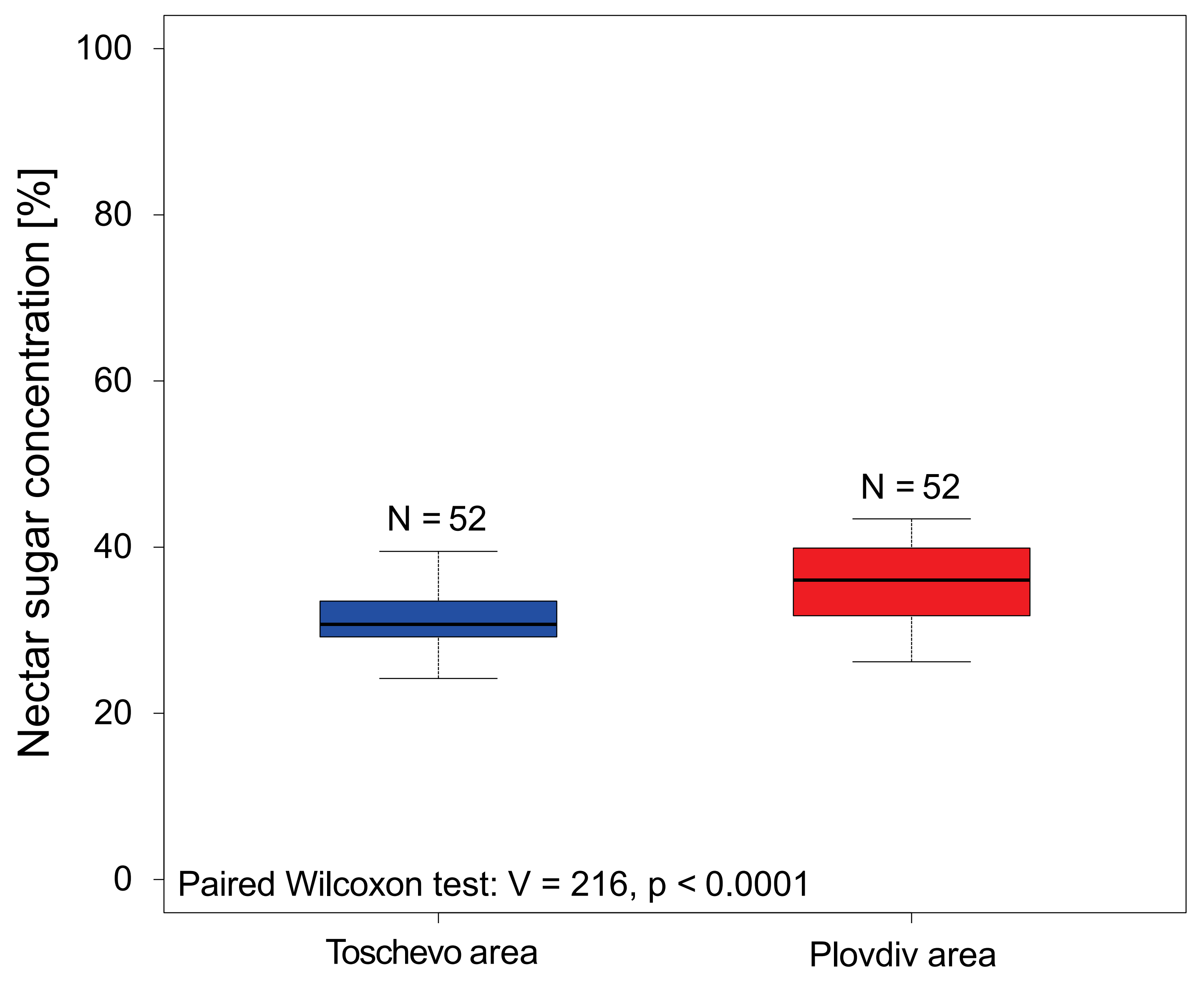




\section{Figure 3 (on next page)}

Nectar sugar concentration on a genus level

Shows the distribution of nectar sugar concentration among all plant genera for which more than 3 measurements were available. The red lines indicate the boarder of the optimal (65-35\%), adequate (35$20 \%$ ) and low sugar nectar concentration (<20\%). Crop genera are marked in red. Results of the statistical analysis (Kruskal Wallis (KW) $\mathrm{chi}^{2}$, and $\mathrm{p}$ values) are presented in the lower left Corner. 


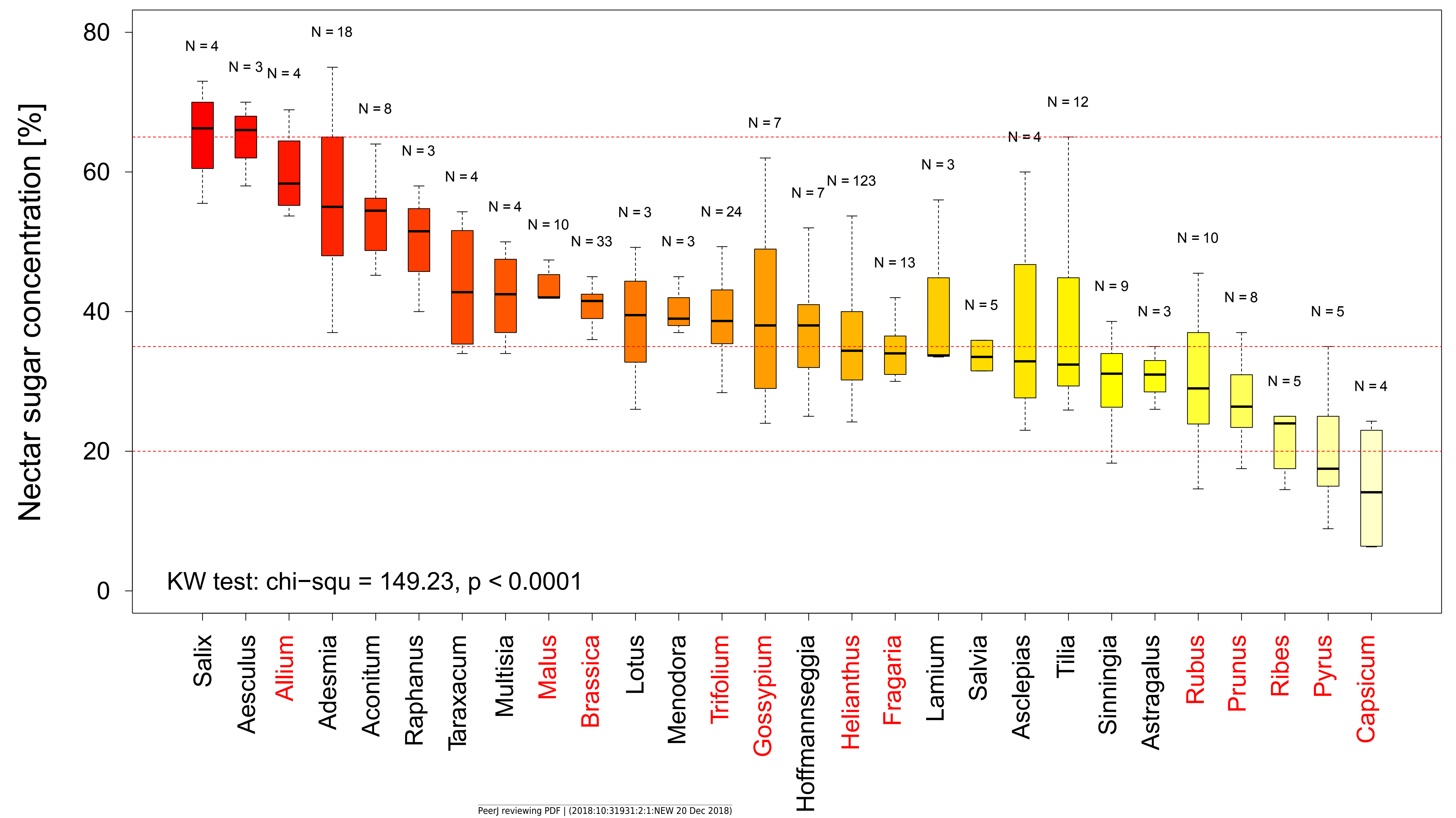




\section{Table $\mathbf{1}$ (on next page)}

Summary statistics of nectar sugar concentrations

Summary statistic of the sugar concentration [\%] of crop, weed and wild plant communities across the globe 
Table 1.: Summary statistic of the sugar concentration [\%] of crop, weed and wild plant communities across the globe

\begin{tabular}{|c|c|c|c|c|c|c|}
\hline Region & Community & $\mathrm{N}$ & median & mean & 10th Percentile & 25th Percentile \\
\hline \multirow[t]{4}{*}{ Global } & ALL & 322 & 40 & 41.0 & 25 & 32 \\
\hline & Crop & 151 & 39.7 & 39.2 & 24 & 32 \\
\hline & Weed & 30 & 39.8 & 41.6 & 30.5 & 33.6 \\
\hline & Wild & 141 & 41 & 32 & 25 & 32 \\
\hline \multirow[t]{4}{*}{ Europe } & ALL & 236 & 39.7 & 40.3 & 24.9 & 32.3 \\
\hline & Crop & 144 & 39.9 & 39.4 & 24.1 & 32.9 \\
\hline & Weed & 30 & 39.8 & 41.6 & 30.5 & 33.6 \\
\hline & Wild & 62 & 39.3 & 41.7 & 24.9 & 29.9 \\
\hline \multirow[t]{4}{*}{ North America } & ALL & 240 & 40 & 40.9 & 25 & 32.7 \\
\hline & Crop & 145 & 40 & 39.6 & 24.1 & 33 \\
\hline & Weed & 30 & 39.8 & 41.6 & 30.5 & 33.6 \\
\hline & Wild & 65 & 44 & 43.8 & 25.3 & 32.3 \\
\hline \multirow[t]{4}{*}{ South America } & ALL & 234 & 40 & 41.3 & 26.4 & 33.9 \\
\hline & Crop & 136 & 40 & 40.8 & 28.3 & 34.9 \\
\hline & Weed & 18 & 41.7 & 42.5 & 32.9 & 37 \\
\hline & Wild & 80 & 40 & 42 & 26 & 32 \\
\hline \multirow[t]{4}{*}{ Africa } & ALL & 168 & 41 & 41.9 & 27.5 & 34.9 \\
\hline & Crop & 133 & 40 & 40.9 & 28.5 & 35 \\
\hline & Weed & 13 & 40 & 42.8 & 32.8 & 34 \\
\hline & Wild & 22 & 51.8 & 47.6 & 23.3 & 34 \\
\hline \multirow[t]{4}{*}{ Asia } & ALL & 211 & 40 & 40.9 & 26 & 33 \\
\hline & Crop & 141 & 40 & 40 & 25.8 & 34 \\
\hline & Weed & 25 & 43.4 & 42.5 & 31.7 & 34.7 \\
\hline & Wild & 45 & 40 & 42.9 & 25.4 & 32.3 \\
\hline \multirow[t]{4}{*}{ Australia } & ALL & 203 & 40 & 40.9 & 26 & 33 \\
\hline & Crop & 139 & 40 & 40 & 25.8 & 43 \\
\hline & Weed & 24 & 39.8 & 41.6 & 31.4 & 33.4 \\
\hline & Wild & 40 & 40.8 & 43.6 & 25.9 & 32.5 \\
\hline
\end{tabular}


